\title{
Growth of Serpentine Carbon Nanotubes on Quartz Substrates and Their Electrical Properties
}

\author{
Seokwoo Jeon ${ }^{1,2,3}$, Changgu Lee ${ }^{2,3}$, Jinyao Tang ${ }^{1,3}$, James Hone $^{2,3}(\bowtie)$, and Colin Nuckolls ${ }^{1,3}(\bowtie)$ \\ ${ }^{1}$ Department of Chemistry, Columbia University, New York, NY 10027, USA \\ ${ }^{2}$ Department of Mechanical Engineering, Columbia University, New York, NY 10027, USA \\ ${ }^{3}$ The Columbia University Nanoscale Science and Engineering Center, Columbia University, New York, NY 10027, USA \\ Received: 29 August 2008/ Revised: 25 September 2008/ Accepted: 25 September 2008 \\ CTsinghua Press and Springer-Verlag 2008. This article is published with open access at Springerlink.com
}

\begin{abstract}
A simple method for high-yield, chemical vapor deposition (CVD) synthesis of serpentine carbon nanotubes, employing quartz substrates and a molecular cluster catalyst, is described. The growth mechanism is analyzed by controlled addition of nanoscale barriers, and by mechanical analysis of the curved sections. The serpentine structures are used to study the electrical transport properties of parallel arrays of identical nanotubes, which show three-terminal conductance that scales linearly with the number of nanotube segments.
\end{abstract}

\section{KEYWORDS}

Aligned carbon nanotube, chemical vapor deposition growth, quartz substrate, electronics

Carbon nanotubes (CNTs) are exceptional materials that possess superior electrical properties and chemical functionalities [1, 2]. Their small diameter and mechanical robustness render them useful for flexible and wearable electronics applications [35]. Recent studies using chemical vapor deposition (CVD) growth, using substrates such as sapphire $[6,7]$ and quartz [8], have demonstrated highly aligned CNTs over large areas, limited only by the size of substrates and the CVD chamber. Compared to sapphire, quartz substrates offer a much cheaper alternative that has the added advantage of having a more stable template along its [1120] step edge direction when the (1101) surface is exposed [8]. The growth gives dense, template assisted CNTs in a "wake-growth" mechanism where the metal nanoparticles move in parallel to the step edge and leave the CNTs behind [9]. The average density and length of the aligned CNT depend on metal catalyst and growth parameter.

There are reports that template assisted growth of CNTs is not affected by the direction of the carbon feedstock flow gas $[8,9]$. It is incongruous that the CNT growth would have the extreme sensitivity of step height to growth, but not to the direction of gas flow. In fact, we have observed various shapes of CNTs (serpentine, loop, etc.) when the quartz step edge direction does not parallel to the flow direction. Here we detail our studies of template assisted growth of serpentine CNTs and electrical measurements from those tubes. The method of making the serpentine CNTs is simple and the yield

Address correspondence to Colin Nuckolls, cn37@columbia.edu; James Hone, jh2228@columbia.edu 
of long, dense, and serpentine tubes is high. A typical example is shown in Fig. 1. This method, combined with transfer techniques [3] to apply the CNTs to various substrates, such as $\mathrm{Si}, \mathrm{Si}_{3} \mathrm{~N}_{4}$, and glass, provides an alternative and advantageous method to create, study, and utilize these novel structures that does not require contact mode AFM [10, 11]. Very recently, two independent groups similarly observed a serpentine type of CNT growth on sapphire and quartz $[12,13]$. To increase the yield of serpentine CNTs, they first patterned $10-30 \mathrm{~nm}$ thick islands or stripes of amorphous $\mathrm{SiO}_{2}$ on a quartz substrate and then deposited ferritin or evaporated Fe on top. They showed linear dependence between the diameter and bending radius of CNTs and the measured conductance with two terminal devices. In this work, we use a different metal catalyst that provides a simpler method of growth with higher yield, does not require any raised islands or stripes, and utilizes a much lower (less than 1/10) flow rate of feedstock gas. The mechanism for growth is elucidated by changing catalysts and examining the pattern of growth across added thin $(\sim 1 \mathrm{~nm})$ stripes of $\mathrm{HfO}_{2}$. Also, the narrowly distributed value of the bending moment, calculated from the diameter and bending radius of the CNTs, may give some hint about the growth mechanism. Finally, two- and three-terminal electrical transport measurements on parallel segments of the serpentine tubes show the usefulness of these tubes for applications in electrical devices.

The metal nanoclusters used for the CNT growth in our experiment are a supramolecular compound known as the Müller catalyst, which is a Fe and Mo complex caging a Keggin ion at the core [14]. This homogenous compound provides metal nanodots (diameter $=1.3 \mathrm{~nm} \pm 0.5 \mathrm{~nm}$ ) after annealing [15]. Its high solubility in polar solvents helps to form uniform nanodots without aggregation after wet deposition. The Müller catalyst produces CNTs with a narrow distribution of diameters (centered at $1 \mathrm{~nm}$ ) [15]. Moreover, we show here that this catalyst is exceptionally efficacious at growing serpentine type CNTs. Dilute Müller catalyst in ethanol $\left(10^{-7}-10^{-8}\right.$ $\mathrm{mol} / \mathrm{L}$ ) consistently produces dense serpentine CNTs without the need for raised platforms. When we use ferric chloride or e-beam evaporated metal (Fe or Co),

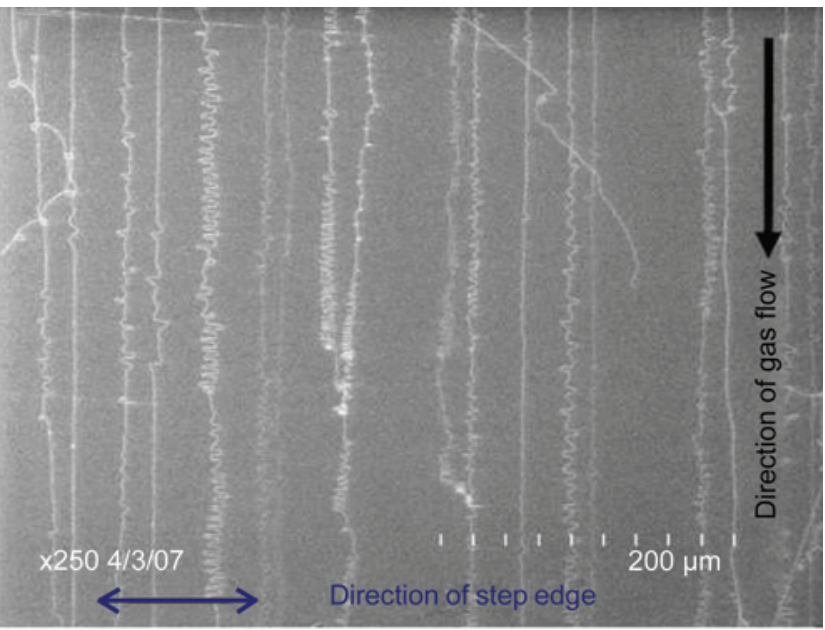

(a)

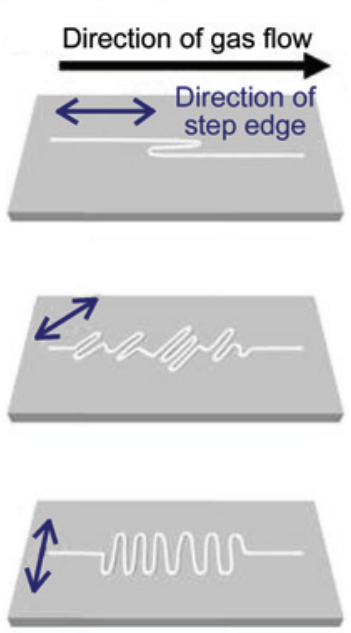

(b)

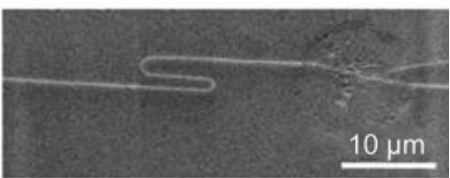

(c)

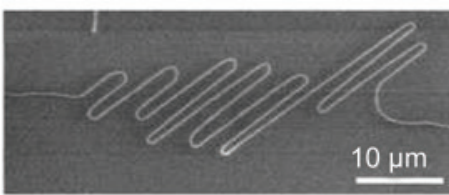

(d)

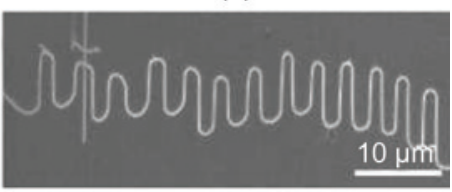

(e)
Figure 1 (a) SEM image of long and dense serpentine CNTs grown on a quartz surface and (b) schematic illustrations showing the variation of tendency in serpentine growth when the angle between the direction of feedstock gas flow and step edge is $0^{\circ}$ (top frame), $45^{\circ}$ (middle frame), and $90^{\circ}$ (bottom frame); (c)-(e) SEM images of serpentine tubes corresponding to the schematics in (b). All samples in this figure are grown at the same flow rate of $\operatorname{Ar}(20 \mathrm{sccm})$ and $\mathrm{H}_{2}$ (10 sccm) with the Müller catalyst

the density of serpentine tubes is lower, and their quality (length, number of turns, etc.) is poorer than the tubes grown from the Müller catalyst. The use of the Müller catalyst makes the overall growth process simpler and may be the reason for the lower flow rate (20 and $10 \mathrm{sccm}$ of $\mathrm{Ar}$ and $\mathrm{H}_{2}$, respectively) of the carbon feedstock compared to that in Geblinger's studies (250 to $2000 \mathrm{sccm}$ of $\mathrm{Ar}$ and $\mathrm{H}_{2}$ combined) [12].

Figure 1 shows micrographs that clearly correlate the direction of the carrier gas and the orientation of the step edges with the growth tendency of serpentine tubes. We first prepare the quartz substrates by 
cleaving them from a wafer (Hoffman Materials, Inc.) to the desired orientation and annealing them in air at $900{ }^{\circ} \mathrm{C}$ for $\sim 8 \mathrm{~h}$. The catalyst solution is quickly spread at one edge of the quartz substrate with a cotton swab and the sample is placed in a 1-inch quartz tube for oxidation for one hour at $500{ }^{\circ} \mathrm{C}$. After purging with Ar, a mixture of $300 \mathrm{sccm} \mathrm{Ar}$ and $100 \mathrm{sccm} \mathrm{H}_{2}$ reduces the nanoparticles at $750{ }^{\circ} \mathrm{C}$ for $1 \mathrm{~h}$. Once the temperature is raised to $880{ }^{\circ} \mathrm{C}$, we pass $20 \mathrm{sccm}$ of Ar and $10 \mathrm{sccm}$ of $\mathrm{H}_{2}$ through an ethanol bubbler in an ice bath to supply the carbon source for the CNT growth for $\sim 30 \mathrm{~min}$. Figure 1(a) shows a typical scanning electron micrograph (SEM) from such a growth. Many of the CNTs are longer than $3 \mathrm{~mm}$ and contain both straight and serpentine segments. Depending on the angle between the direction of gas flow and the step edge of substrate $\left(0^{\circ}\right.$ for Fig. 1(c), $45^{\circ}$ for Fig. 1(d), and $90^{\circ}$ for Fig. 1(e)), the shape and growth tendency of the serpentine CNTs are significantly changed. More than 50 continuous turns of serpentine CNTs are frequently observed from $90^{\circ}$ samples, but it is rare to find more than 20 turns of continuous, regularly spaced ones from $45^{\circ}$ samples and more than 2 turns from $0^{\circ}$ samples. Also, the length of straight segments before the turns are $~ 5-15$ $\mu \mathrm{m}$ for $90^{\circ}$ samples and $\sim 10-30 \mu \mathrm{m}$ for $45^{\circ}$ samples. These results strongly imply that growth mechanism is related to the flow of feedstock gas.

Geblinger and coworkers propose a "falling spaghetti" [12] growth mechanism for the serpentine tubes. In this mechanism, the CNT is tip-grown and becomes buoyant over the substrate along the direction of gas flow, which induces tension in the suspended CNT. After approaching a certain critical length, the CNT falls and initiates a rapid oscillatory motion, providing a competition between the flowinduced tension and the adhesion between the CNT and substrate. The morphology of some of the serpentines supports this mechanism. The straight segments of the serpentine tubes become wider, but the spacing of the straight segments becomes narrower along the direction of growth. The "falling spaghetti" mechanism explains this behavior because compared to the initial length of the buoyant CNTs, the shorter length of the suspended CNTs during serpentine motion decreases the tension from the gas flow. Considering a laminar flow on the substrate, the loss of tension would be very fast at the end. We also frequently observe this correlation in our experiments, but as can be seen in Fig. 1 it is not universal. Because of this apparent inconsistency, we report here our investigation into the mechanism of the growth.

Tension from the buoyant part of CNTs during growth is a key to explain the mechanism of growth, and in particular explains why the serpentine CNTs always curve toward the flow direction. The turns of Fig. 1(c), are rather unexpected when the $\mathrm{CNT}$ is in tension toward the flow direction, but possible if the flow inside the quartz tube is not in steady state and the falling of the CNT is a fast, non-equilibrium phenomenon. It is also important to note that such turns are never observed from template assisted growth of dense, aligned CNTs [8, 9], which typically uses e-beam evaporated $\mathrm{Fe}$ or $\mathrm{Co}$, indicating that catalyst concentration can affect the growth mechanism. The effects of catalyst density are shown explicitly in Fig. 2(a). CNTs in this sample are grown by the catalysis of a 1:10 solution of $\mathrm{FeCl}_{3}$ and ethanol. The diagonal white stripe in Fig. 2(a) is where ferric chloride is in high concentration due to aggregation during drying. Growth from the region of the concentrated catalyst is extremely straight and dense, similar to highly aligned tubes from a "wakegrowth mechanism" $[8,9]$. The growth of serpentine CNTs is mostly initiated from locations where catalyst is sparse and isolated. This observation explains why one cannot find the effect of flow from normal aligned growth and such turns in Fig. 1(c). A well spaced catalyst such as the Müller catalyst does not make CNTs bundle and seems to promote the buoyancy of tip-grown tubes which is the initial step of the "spaghetti falling mechanism".

To further understand the growth mechanism, we fabricated barriers on a quartz surface that are high enough to discourage growth parallel to the step edge but low enough not to affect the tip-growth of buoyant CNTs. $\mathrm{HfO}_{2}$ stripes with a width of $50 \mu \mathrm{m}$ and height of $1 \mathrm{~nm}$, and $150 \mu \mathrm{m}$ pitch, were deposited parallel to the direction of the step edge through atomic layer deposition. If the growth of mechanism serpentines were solely from wake-growth, all the serpentine would stop growing at the barrier. Interestingly, the 


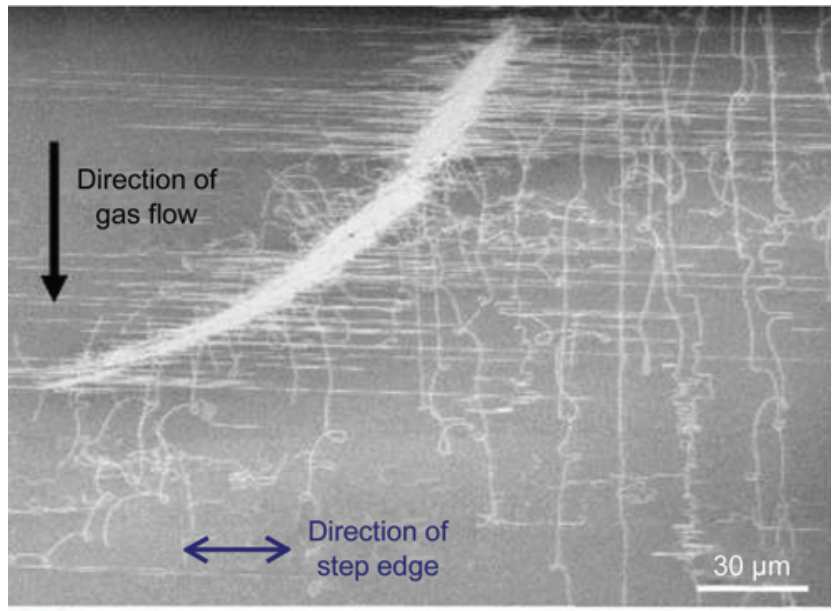

(a)

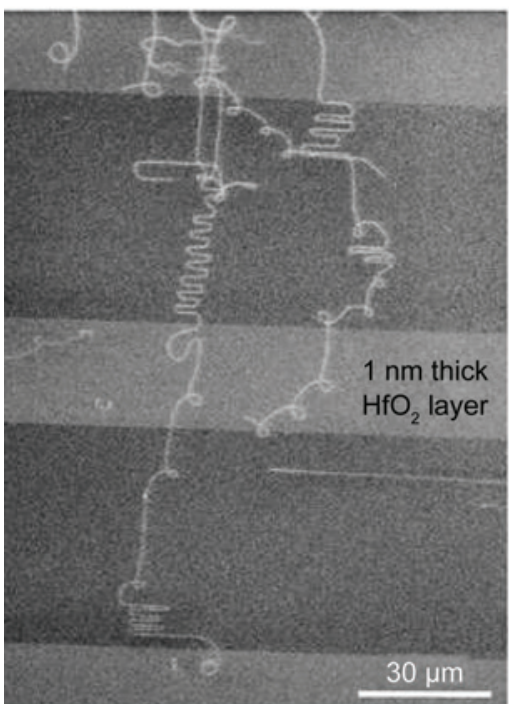

(b)

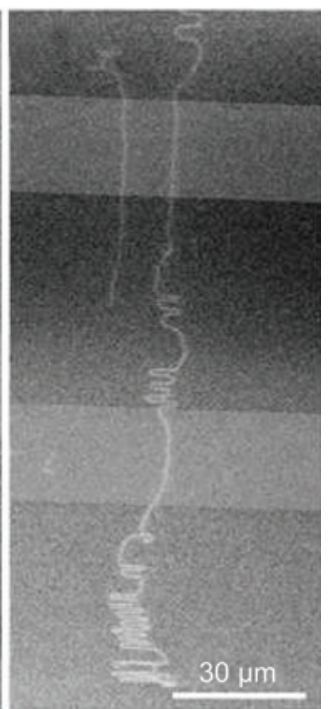

(c)
Figure 2 (a) SEM image of CNTS grown from ferric chloride showing two distinctive modes of growth: highly straight growth along the direction of step edge and erratic growth along the direction of gas flow; (b), (c) SEM images of serpentine growth over the barrier of 1 $\mathrm{nm}$ thick $\mathrm{HfO}_{2}$ (gray stripes). A serpentine tube loses its motion of lateral undulation on the barrier (center stripe in (b)), or goes over the barrier and resumes serpentine movement (c)

CNTs grow over the $\mathrm{HfO}_{2}$ layer. Figure 2(b) shows that a segment of serpentine loses its track at the boundary and Fig. 2(c) shows that serpentine tubes become straight on top of the $\mathrm{HfO}_{2}$ and then resume lateral undulation again. This behavior is strong support for the "spaghetti falling mechanism" for serpentine CNTs. Another observation, which is consistent with the work of Geblinger and coworkers [12], is that higher flow rates (>100 sccm) under our growth conditions reduce the yield of serpentines. However, control experiments that change the speed of gas flow after closing the feedstock bubbler seemingly do not affect the yield or overall shape of serpentine growth, in contrast to their observation.

Figure 3 presents results of atomic force microscopy (AFM) and SEM measurements of the CNT diameter $(d)$ and the bending radius $(R)$. As expected, we observe that the larger diameter CNTs give larger curvatures. More interestingly, the derived values of $d^{3} / 2 R$, which is proportional to the internal bending moment of a curved cylindrical beam, are similar for many of the serpentines. The values of $d^{3} / 2 R$ are $\sim 2$ when the diameters of CNT are smaller than $2 \mathrm{~nm}$ (Table 1). However, the values jump to $\sim 7$ when the diameter is larger than $2 \mathrm{~nm}$. This may imply the latter tubes are multi-wall tubes. The calculated energy to bend a floating CNT is $\sim 1000$ times smaller $(\sim 1 \mathrm{meV} / \mathrm{nm}, d=1.4 \mathrm{~nm})$ than the van der Waals energy $(2 \mathrm{eV} / \mathrm{nm}, d=1.4 \mathrm{~nm})$ between the quartz substrate and the CNT [12]. We see similar behaviors when we measure the quartz surface with force displacement measurement using AFM. This fact again supports our observation that no serpentines, nor even a single turn, can be found from the growth modes of dense, aligned CNTs in Fig. 2(a); non-buoyant wake-growth tubes cannot overcome the strong adhesion energy to derail. From this, one may assume the internal bending moment plays a key role in determining the value of the bending radius. However, another interesting observation is the change of serpentine frequency even in a single tube. The change of serpentine frequency is abrupt not

Table 1 Table of measured diameter (d) of CNTs from AFM and radius of bending $(R)$ from SEM. Data for Sample 3 are measured from images of Fig. 3(a) and data of smaller bending radius in Sample 6 are from images of Fig. 3(b). Figure 3(c) corresponds to Sample 7. The bending radius obtained by SEM is the average value of more than 3 adjacent turns, and the diameter of CNT obtained by AFM is the average value of height measurement from more than 3 adjacent CNTs

\begin{tabular}{c|c|c|c}
\hline Sample & $d(\mathrm{~nm})$ & $2 R(\mu \mathrm{m})$ & $d^{3} /(2 R)$ \\
\hline 1 & 1.2 & 0.878 & 1.97 \\
\hline 2 & 2.5 & 2.17 & 7.20 \\
\hline 3 & 2.6 & 2.5 & 7.03 \\
\hline 4 & 1.6 & 1.53 & 2.68 \\
\hline 5 & 1.2 & 0.862 & 2.00 \\
\hline 6 & $1.2 / 1.2$ & $0.835 / 1.24$ & $2.07 / 1.39$ \\
\hline 7 & $1.3 / 1.3$ & $1.03 / 1.32$ & $2.13 / 1.66$ \\
\hline
\end{tabular}




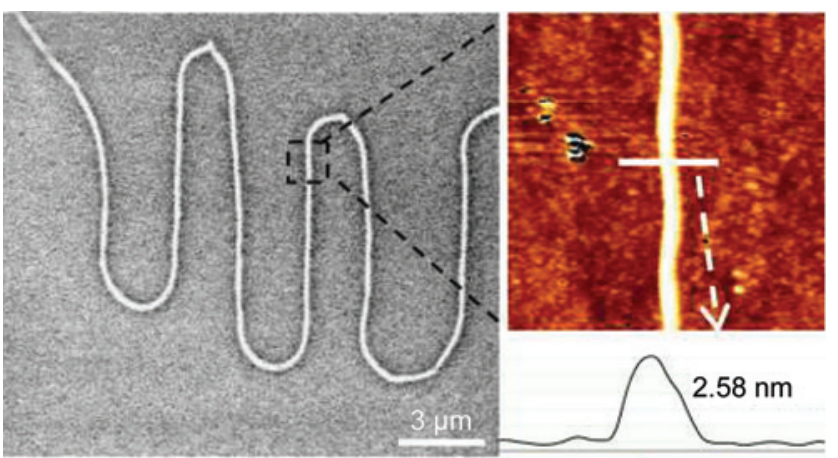

(a)

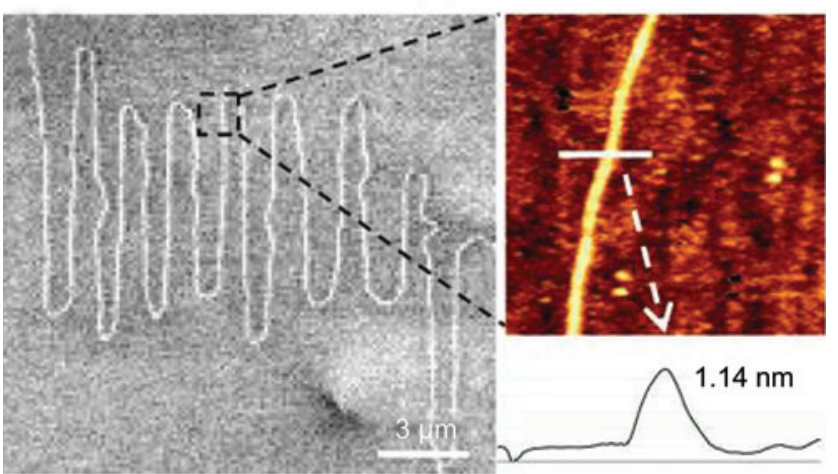

(b)

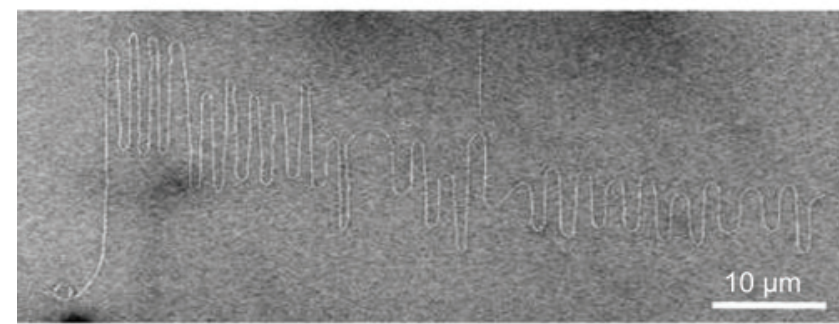

(c)

Figure 3 (a), (b) Representative SEM and AFM images of serpentine tubes with large $(>2 \mu \mathrm{m})$ and small $(<1 \mu \mathrm{m})$ diameter of bending; (c) SEM of CNT which significantly changes its bending diameter from 1.32 to $1.03 \mu \mathrm{m}$ (from right to left)

gradual, as shown in Fig. 3(c). The left part has higher frequency of serpentine movement, and the right part has lower frequency. The sections have similar tube diameter within experimental error but differ in radius of curvature (Sample 7 in Table 1), by $~ 30 \%$; even still, the value of the curvature does not affect the value of $d^{3} /(2 R)$ much as do the values for larger diameter CNTs (Samples 2 and 3 in Table 1). These observations mean that the internal bending moment has a certain contribution to the radius of curvature, but is not the determining factor because of nonsteady nature of the serpentine growth as reported by Geblinger et al. [12].

The serpentines and rings shown in this paper can be useful for the fabrication of nanoelectronic components such as resistive heaters, resonant antennae, and many others if they can be transferred at specific locations on selected substrates. In addition, these structures allow us to address fundamental questions of device scaling and design. Specifically, because the theoretical limit of conductance through a single nanotube is low due to quantum resistance of CNTs ( $\sim 6 \mathrm{k} \Omega /$ tube), many useful CNT devices will require parallel arrays of nanotubes [16] to minimize the device impedance. However, it has not been experimentally verified that such arrays can be treated as a simple parallel circuit in which the conductances are simply added.

The inset to Fig. 4 shows three two-terminal devices consisting of multiple sections of a single serpentine $\mathrm{CNT}$, fabricated directly on the quartz substrate by electron beam lithography and liftoff of Pd. As shown in Fig. 4, the device conductance scales linearly with the number of nanotube sections between the leads, with a constant two-terminal resistance of $\sim 300 \mathrm{k} \Omega /$ tube for all three devices.

To examine the three-terminal behavior of these structures, serpentine CNTs were first transferred to a degenerately-doped silicon wafer with a $300-$ $\mathrm{nm} \mathrm{SiO}_{2}$ epilayer, followed by device fabrication as described above (Fig. 5, inset). The transfer process consists of mechanically peeling off thin films of CNTs supported by $\sim 50 \mathrm{~nm}$ gold and a flexible water soluble polymer (PVA), contacting it to the Si wafer,

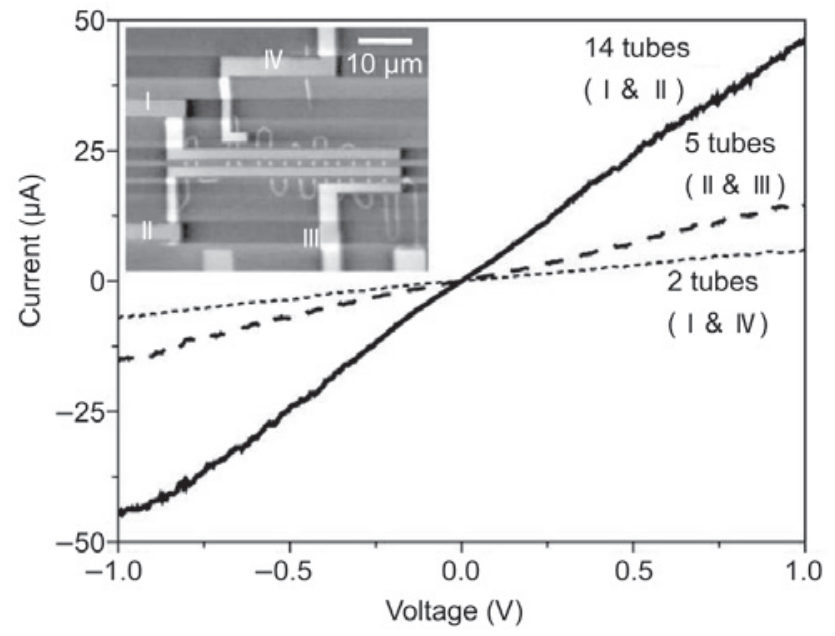

Figure $4 \quad I-V$ plots from two-terminal devices made over a serpentine tube on a quartz substrate. Three different numbers $(2$, 5 , and 14) of CNTs are bridging four contact leads (I, II, III, and IV) as shown in the inset image

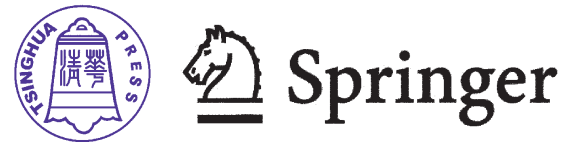


and etching away the polymer and gold (details of the transfer method can be found elsewhere $[3,5]$ ). Figure 5 shows a plot of the current vs gate voltage for three devices, with 5, 10, and 13 parallel sections, at a constant bias of $10 \mathrm{mV}$. All three devices show p-type semiconducting behavior, with good on-off ratio $\left(>10^{4}\right)$, turn-on voltage around $2.5 \mathrm{~V}$, and typical hysteresis between up- and down-sweeps [17]. Figure 5(b) shows the conductance per nanotube segment for all three devices. The 5- and 13-tube devices show virtually identical behavior (the estimated value of mobility [5] from these devices are $1000 \mathrm{~cm}^{2} /(\mathrm{V} \cdot \mathrm{s})$ ).

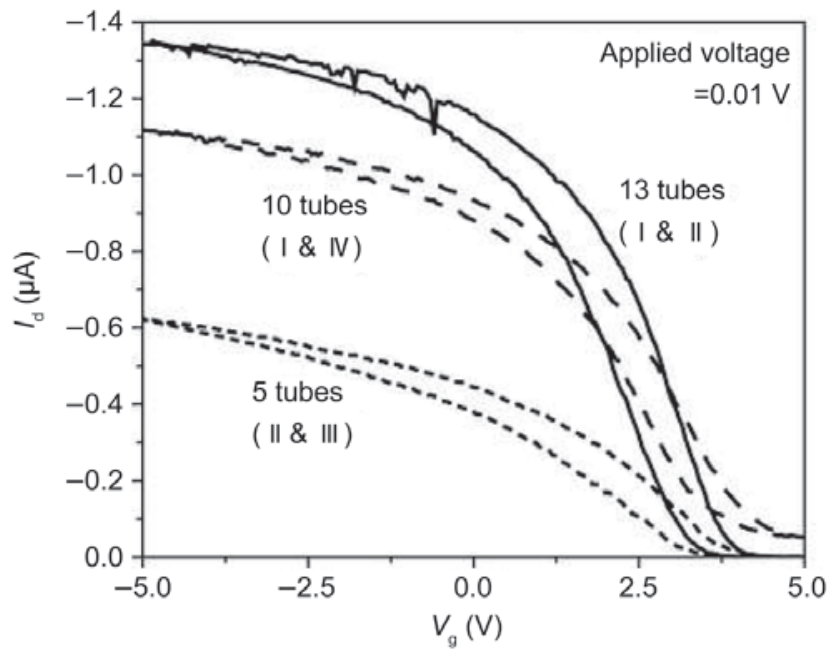

(a)

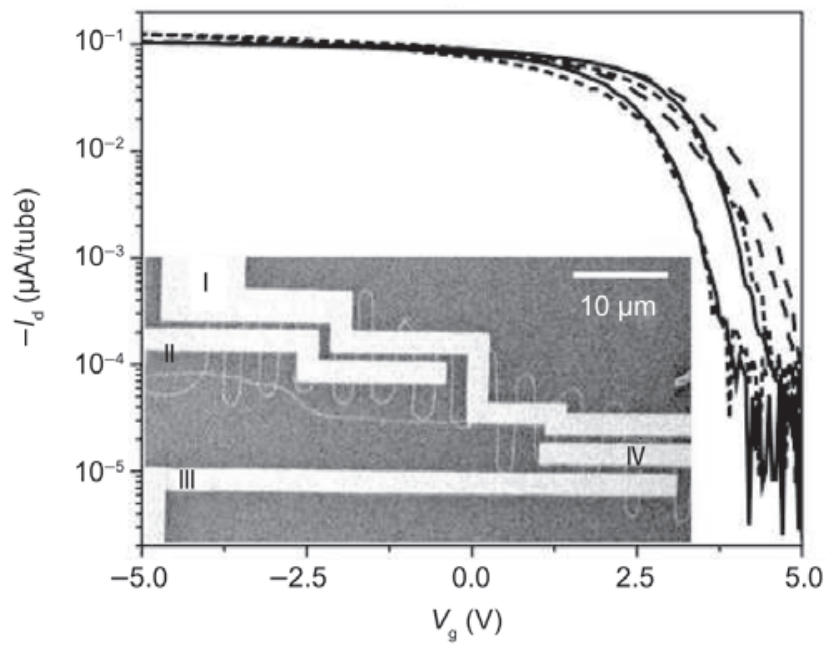

(b)

Figure 5 (a) Plot of gate sweep under $0.01 \mathrm{~V}$ of applied source to drain voltage from a back gate device. Three different numbers (5, 10, and 13) of CNTs are connected to four contact leads (I, II, III, and IV) as shown in the inset image of (b). The device mades of 10 bridged tubes cannot be turned off completely. (b) Normalized plot of (a) per tube value. Leakage current from the device of 10 bridged tubes is deducted for better comparison
The 10-tube device differs slightly, but this is likely due to the presence of a parasitic conduction channel through a spurious additional tube. The data confirm the expected scaling of device resistance with number of nanotube channels. In addition, the identical subthreshold behavior of the devices shows that all of the nanotube sections possess identical transfer characteristics (i.e., threshold voltage and transconductance), and demonstrates that parallel array CNT-FETs can achieve the same high performance as individual nanotubes. With this method, one can easily get many parallel CNTs with the same chirality and diameter. We have fabricated devices with on-state resistances as low as $\sim 800 \Omega$ in this manner.

In conclusion, combined effects from flow direction of carbon feedstock gas and step direction of quartz substrate can promote growth of various shapes of CNTs and the angles between those directions play an important role in determining the overall shapes of grown CNTs. The use of the Müller catalyst forms uniform and well distributed metal nanoparticles on a quartz surface that enables us to achieve long, high yield serpentine tubes. The dominant growth mechanism of serpentine tubes is not wake-growth, but a combination of buoyant growth and a falling mechanism. The observed narrow distribution of bending moments, obtained from the analysis of bending radius and diameter of the CNTs, supports the proposed growth mechanism. Electrical measurements on CNT arrays show linear scaling of the three-terminal electronic transport with the number of tubes, without any extrinsic broadening or other effects. The level of control over the growth and interesting electronic properties promise this technique will offer a useful tool to characterize electric and mechanical properties of CNTs and to fabricate new CNT-based devices.

\section{Acknowledgements}

This work is supported by the Nanoscale Science and Engineering Initiative of the National Science Foundation under NSF Award Number CHE-0117752 and by the New York State Office of Science, Technology, and Academic Research (NYSTAR). 


\section{References}

[1] Jorio, A.; Dresselhaus, M. S.; Dresselhaus, G. Carbon Nanotubes; Springer: New York, 2008.

[2] O'Connell, M. J. Carbon Nanotubes: Properties and Applications; CRC Press: Boca Raton, 2006.

[3] Ahn, J. -H.; Kim, H. -S.; Lee, K. J.; Jeon, S.; Kang, S. J.; Sun, Y.; Nuzzo, R. G.; Rogers, J. A. Heterogeneous threedimensional electronics by use of printed semiconductor nanomaterials. Science 2006, 314, 1754-1757.

[4] Dondero, W.; Gorga, R. E. Carbon nanotubes. In Handbook of Nanoscience, Engineering, and Technology, 2nd ed.; Goddard, W. A.; Brenner, D. W.; Lyshevski, S. E.; lafrate, G. J., Eds; CRC Press: Boca Raton, 2007, pp. 21/27-21/37.

[5] Kang, S. J.; Kocabas, C.; Ozel, T.; Shim, M.; Pimparkar, N.; Alam, M. A.; Rotkin, S. V.; Rogers, J. A. Highperformance electronics using dense, perfectly aligned arrays of single-walled carbon nanotubes. Nature Nanotech. 2007, 2, 230-236.

[6] Ago, H.; Nakamura, K.; Ikeda K. -l.; Uehara, N.; Ishigami, $\mathrm{N}$.; Tsuji, M.; Aligned growth of isolated single-walled carbon nanotubes programmed by atomic arrangement of substrate surface. Chem. Phys. Lett. 2005, 408, 433438.

[7] Ismach, A.; Kantorovich, D.; Joselevich, E. Carbon nanotube graphoepitaxy: Highly oriented growth by faceted nanosteps. J. Am. Chem. Soc. 2005, 127, 1155411555.

[8] Kocabas, C.; Hur, S. -H.; Gaur, A.; Meitl, M. A.; Shim M.; Rogers, J. A. Guided growth of large-scale, horizontally aligned arrays of single-walled carbon nanotubes and their use in thin-film transistors. Small 2005, 1, 11101116.

[9] Ding, L.; Yuan, D.; Liu J. Growth of high-density parallel arrays of long single-walled carbon nanotubes on quartz substrates. J. Am. Chem. Soc. 2008, 130, 5428-5429.

[10] Hertel, T.; Martel, R.; Avouris, P. Manipulation of individual carbon nanotubes and their interaction with surfaces. J. Phys. Chem. B 1998, 102, 910-915.

[11] Park J. -Y. Carbon nanotube field-effect transistor with a carbon nanotube gate electrode. Nanotechnology 2007, 18, 095202.

[12] Geblinger, N.; Ismach, A.; Joselevich, E. Self-organized nanotube serpentines. Nature Nanotech. 2008, 3, 195200.

[13] Kocabas, C.; Kang, S. J.; Ozel, T.; Shim, M.; Rogers, J. A. Improved synthesis of aligned arrays of single-walled carbon nanotubes and their implementation in thin film type transistors. J. Phys. Chem. C 2007, 111, 1787917886.

[14] Müller, A.; Das, S. K.; Kögerler, P.; Bögge, H.; Schmidtmann, M.; Trautwein, A. X.; Schunemann, V.; Krickemeyer, E.; Preetz, W. A new type of supramolecular compound: Molybdenum-oxide-based composites consisting of magnetic nanocapsules with encapsulated Keggin-ion electron reservoirs cross-linked to a twodimensional network. Angew. Chem., Int. Ed. Engl. 2000, 39, 3414-3417.

[15] An, L.; Owens, J. M.; McNeil, L. E.; Liu, J. Synthesis of nearly uniform single-walled carbon nanotubes using identical metal-containing molecular nanoclusters as catalysts. J. Am. Chem. Soc. 2002, 124, 13688-13689.

[16] Keshavarzi, A.; Raychowdhury, A.; Kurtin, J.; Roy, K.; De, $V$. Carbon nanotube field-effect transistors for high-performance digital circuits-transient analysis, parasitics, and scalability. IEEE Trans. Electron Devices 2006, 53, 2718-2726.

[17] Lee, J. S.; Ryu, S.; Yoo, K.; Choi, I. S.; Yun, W. S.; Kim, J. Origin of gate hysteresis in carbon nanotube field-effect transistors. J. Phys. Chem. C 2007, 111, 12504-12507. 